\title{
COVID-19 and Iranian Medical Students; A Survey on Their Related-Knowledge, Preventive Behaviors and Risk Perception
}

\author{
Mohammad Hossein Taghrir, MD ${ }^{1}$; Roham Borazjani, MD ${ }^{1 *}$; Ramin Shiraly, MD² \\ ${ }^{1}$ Medical Student at Shiraz University of Medical Sciences, Student research committee, Shiraz University of Medical Sciences, Shiraz, Iran \\ ${ }^{2}$ Associate Professor of Community Medicine, Department of Community Medicine, School of Medicine, Shiraz University of Medical \\ Sciences, Shiraz, Iran
}

\begin{abstract}
Background: Since December 2019, a novel coronavirus disease (COVID-19) began its journey around the world. Medical students, as frontline healthcare workers, are more susceptible to be infected by the virus. The aim of this study was to assess COVID-19 related knowledge, self-reported preventive behaviors and risk perception among Iranian medical students within the first week after the onset of the outbreak in Iran.

Methods: This cross-sectional study was conducted from 26th to 28th of February, 2020. Participants were Iranian medical students (5th-7th year) whose knowledge, preventive behaviors and risk perceptions of COVID-19 were assessed using an online questionnaire. The questionnaire consisted of 26 questions including 15 items about COVID-19 related knowledge, 9 items regarding preventive measures and 2 items about COVID-19 risk perception. The validity and reliability of the questionnaire were shown to be satisfactory.

Results: A total of 240 medical students completed the questionnaire. The mean age of participants was 23.67 years. The average of correct answers of knowledge was $86.96 \%$; and $79.60 \%$ had high level of related knowledge. The average rate of practicing preventive behaviors was $94.47 \%$; and $94.2 \%$ had high level of performance in preventive behaviors. The cumulative score of risk perception was 4.08 out of 8 which was in moderate range. Risk perception was significantly different between stagers and interns and between those being trained in emergency room (ER) and non-ER wards. There was a significant negative correlation between preventive behaviors and risk perception.

Conclusion: We found a high level of COVID-19 related knowledge and self-reported preventive behaviors and moderate risk perception among Iranian medical students.

Keywords: COVID-19, Health behavior, Knowledge, Medical students, Prevention and control, Risk

Cite this article as: Taghrir MH, Borazjani R, Shiraly R. COVID-19 and Iranian medical students; a survey on their relatedknowledge, preventive behaviors and risk perception. Arch Iran Med. 2020;23(4):249-254. doi: 10.34172/aim.2020.06.
\end{abstract}

Received: March 9, 2020, Accepted: March 20, 2020, ePublished: April 1, 2020

\section{Introduction}

In the last month of 2019, an outbreak of viral pneumonia of unknown etiology occurred in Wuhan city in the east of China. ${ }^{1}$ Gene sequencing revealed that the underlying pathogens were enveloped positive-stranded RNA viruses that belong to the family Coronaviridae and the order Nidovirales. ${ }^{2}$ the Coronavirus Study Group (CSG) of the International Committee on Taxonomy of Viruses named it as $2019-\mathrm{nCoV}$ and then changed it to severe acute respiratory syndrome coronavirus 2 (SARS-CoV-2). ${ }^{3}$ On February 11, 2020, the World Health Organization (WHO) designated a new name for the epidemic disease caused by this virus - coronavirus disease 2019 (COVID-19). The most important transmission route that is currently agreed upon, is human-to-human via respiratory droplets or direct contacts. ${ }^{4}$

Like other viruses of the Coronaviridae family, the main clinical manifestations of the disease are fever which occurs in $99 \%$ of the affected persons, dry cough, dyspnea and bilateral patchy infiltration on imaging. ${ }^{4,5}$ The overall mortality rate of COVID-19 is $2 \%$ which is much lower than that of the Severe Acute Respiratory Syndrome (SARS) and Middle East Respiratory Syndrome (MERS). The virus caused an epidemic in China; also, plenty of cases have been reported in many other countries such as the United States, Korea, Italy, and Iran. ${ }^{6}$

COVID-19 was first detected in two people residing in Qom, central Iran on February 19, 2020 according to the Iran's Ministry of Health. ${ }^{7}$ More cases were reported in other cities of Iran including Tehran which is the capital of Iran, Rasht a city in northern Iran, and also in the city of Shiraz, Fars province. The number of confirmed cases is rising day by day mostly because of the increase in the number of laboratories that can perform definite diagnostic test. The last report of laboratory-confirmed COVID-19 cases and deaths in Iran are as follows: until 
March 3, 2020, a total of 2336 cases were confirmed, of whom 77 expired due to COVID-19 and 291 recovered and were discharged from hospitals. ${ }^{8}$

Medical staff have always been at risk of contagious diseases. Knowing that COVID-19 can be probably transmitted even from asymptomatic individuals, the risk is multiplied. ${ }^{4,9,10}$ In a study, the rate of transmission to health professionals was $29 \% .{ }^{11}$ To illustrate the point, one patient, who was scheduled to undergo surgery in Wuhan City, had infected 14 health care workers even before the fever began. ${ }^{12}$

Medical students are the first individuals who may have close contact with the affected people. Lack of proper related knowledge in this population can make them overestimate the situation, increase their stress and anxiety level and may interrupt the appropriateness of their medical judgments. ${ }^{13}$ A basic study of medical students' knowledge of the COVID-19, preventive behaviors and risk perception is necessary since COVID-19 is currently spreading in Iranian hospitals and all over the world. To our knowledge, no study is available yet to assess medical students' COVID-19 related knowledge, preventive behaviors, and risk perception. So, this survey aims to assess these parameters in Iranian medical students.

\section{Materials and Methods}

This cross-sectional study was conducted on Iranian medical students to investigate their COVID-19 related knowledge, self-reported preventive behaviors and risk perception. Stagers and interns in Iran are the first ones who encounter and visit suspicious cases of COVID-19 in university hospitals and in a way, they are the front-line warriors in this combat. Stager is defined as a 5-6th year and intern is defined as a 7 th year medical student in a 7-year medical education curriculum.

The target population was those who are spending their clinical courses in university hospitals all over the country with an estimated population of 5000. The sample size was calculated by Raosoft sample size calculator. Based on the estimated population and response distribution of $50 \%$, the required sample size was 357 with a confidence level of $95 \%$ and $5 \%$ margin of error.

This study was conducted using an online questionnaire in Persian language from 26th to 28th of February, 2020, within a week after the first case of COVID-19 was confirmed in Iran. Due to the specific prevention recommended during the outbreak, including prevention in close contacts and touch precautions, we designed an online questionnaire to follow these recommendations.

\section{Measures}

The questionnaire consisted of four sections: demographic data, COVID-19 related knowledge, self-reported preventive behaviors and risk perception.

\section{Demographics}

Demographic information included age, gender, level of education, the current ward where the student is being trained, and COVID-19 education and source of education. These items were designed based on previous study $^{13}$ and opinion of two expert epidemiologists.

\section{COVID-19 Related-Knowledge}

The level of related knowledge about COVID-19 was assessed in the first section using 15 items based on a framework from previous studies about MERS ${ }^{13,14}$ and a new review on COVID-19. In this section, there were three items about COVID-19 basic science and its etiology, two items about symptoms and incubation period, one item about diagnosis, two items about transmission, four items about public prevention, one item about medical professionals' specific prevention, one item about treatment and one item about referring suspicious cases. The validity of these items was established by a panel of experts including an infectious disease specialist, two expert epidemiologists and two medical interns. A correct answer was assigned 1 point and an incorrect answer or ' $I$ don't know' were assigned 0 points. The total score was converted into a percentile. A score $\geq 75 \%$ was designated as high, $50 \%-75 \%$ as moderate and $\leq 50 \%$ as low level of knowledge. Reliability was tested in a pilot study by 30 participants and in the original study using Cronbach's alpha. The results were alpha $=0.87$ and 0.80 , respectively.

\section{Self-reported Preventive Behaviors}

In order to assess preventive behaviors, nine items were used based on a previous study. ${ }^{13}$ In this section, there were five items were about reducing the use of public places in daily life, one item about preventive behavior during coughing, two items about intensive hand washing and surface disinfection and the last item about talking with people nearby about prevention. Validation of items was established by three experts including one infectious disease specialist and two epidemiologists. Choices were 'yes' or 'no' and the participant was assigned one point for each appropriate behavior and 0 point for inappropriate behaviors. The total score ranged from 0 to 9 and was converted to a percentile. Seventy five percent or higher scores was designated as high performance in preventive behaviors and $<75 \%$ as low performance. Reliability was tested in a pilot study with 30 participants and in the original study using Cronbach's alpha. The results were alpha $=0.72$ and 0.81 , respectively.

\section{Risk Perception}

Based on a previous study, ${ }^{13}$ two items were used to assess risk perception of COVID-19 among participants. Responses were provided using a 4-point Likert-type scale ( 1 = not at all, $4=$ absolutely yes). In order to diminish any bias, the 4-point scale had a shuffled order for participants. 
Validation of items was established by two expert epidemiologists. Total cumulative score ranged from 2 to 8. Scores between 2 to 3 were designated as low, 4 to 5 as moderate and 6 to 8 as high risk perception. Reliability was tested in a pilot study with 30 participants and in the original study using Cronbach's alpha. The results were alpha $=0.8$ and 0.79 , respectively.

\section{Data Analysis}

Data were analyzed using SPSS version 16.0 (IBM, Armonk, NY, USA). We used Kolmogorov-Smirnov to assess normality of distribution of continuous variables; None of the major outcomes followed a normal distribution and were analyzed using the Mann-Whitney test and Spearman's rank correlation test. The frequencies and percentages were computed for categorical variables and the means and standard deviations were calculated for numerical variables.

\section{Results}

A total of 240 medical students responded to the questionnaire, yielding a response rate of $67.22 \%$. The mean age was 23.67 years. Among the respondents, 98 $(40.8 \%)$ were male and $142(59.2 \%)$ were female. The difference between males and females was significant in risk perception at level of $P<0.01$. Females had lower risk perception. Among our participants, 72 (30\%) were interns, who are in direct touch with suspected cases in emergency rooms (ERs). The cumulative score of risk perception was significantly lower in interns compared with stagers at level of $P<0.05$. Participants who being trained in ERs $(36,15 \%)$ had significantly lower risk perception compared with those being trained in other wards such as internal medicine wards and surgery wards at level of $P<0.01$. Less than half (43.3\%) of participants responded that they had received any kind of education about COVID-19. No significant differences were found between participants who had or had not received related education. Those who answered 'Yes" to receiving related education were asked about source of education. Choices were 'WHO, CDC, UpToDate', 'National guidelines', 'Both' and 'Others'. There was no significant relation between them. Other demographic data are demonstrated in Table 1.

Table 2 lists the COVID-19 related knowledge items. The average of correct answers was $86.96 \%$. In this section, $79.6 \%$ of participants had high, $13.8 \%$ had moderate and $6.7 \%$ had low level of related knowledge. The lowest scores pertained to two items; 'If symptoms appear within 14 days from direct contact with a suspected case, the person should inquire at a nearby public health center. (T)' and 'Only during intubation, suction, bronchoscopy and cardiopulmonary resuscitation, you have to wear N95 mask $(\mathrm{T})^{\prime}$.

In the self-reported preventive behaviors section, the average of correct answers was $94.47 \%$. The majority (94.2\%) of participants had high performance in preventive behaviors and only $5.8 \%$ had a low score. The lowest score $(85.6 \%)$ pertained to 'I increased the frequency of cleaning and disinfecting items that can be easily touched with hands (i.e. door handles and surfaces)'. The other items are listed in Table 3.

In the section of risk perception (Table 4), there were two items; 'I may become infected with COVID-19 more easily than others.' and 'I am afraid to be infected with COVID-19'. The mean scores were 2.04 and 2.04, respectively (out of 4). The total cumulative score was 4.08 out of 8 which is indicative of moderate risk perception.

Table 1. Differences in Major Variables According to Demographics, Using Mann-Whitney Test $(\mathrm{N}=240)$

\begin{tabular}{|c|c|c|c|c|c|c|c|c|}
\hline \multirow[t]{2}{*}{ Variables } & \multirow[t]{2}{*}{ Characteristic } & \multirow[t]{2}{*}{ No. $(\%)$} & \multicolumn{2}{|c|}{$\begin{array}{l}\text { Related Knowledge } \\
\text { Range } 0-100\end{array}$} & \multicolumn{2}{|c|}{$\begin{array}{c}\text { Preventive Behaviors } \\
\text { Range } 0-100\end{array}$} & \multicolumn{2}{|c|}{$\begin{array}{l}\text { Risk Perception } \\
\text { Range 2-8 }\end{array}$} \\
\hline & & & Mean \pm SD & $\mathbf{U}$ & Mean \pm SD & $\mathbf{U}$ & Mean \pm SD & $\mathbf{U}$ \\
\hline \multirow{2}{*}{ Gender } & Male & $98(40.8 \%)$ & $87.13 \pm 13.61$ & \multirow{2}{*}{6559} & $91.29 \pm 18.44$ & \multirow{2}{*}{6561} & $4.60 \pm 1.8$ & \multirow{2}{*}{$5176^{* *}$} \\
\hline & Female & $142(59.2 \%)$ & $85.60 \pm 16.57$ & & $95.70 \pm 12.04$ & & $3.72 \pm 1.66$ & \\
\hline \multirow{2}{*}{ Level of education } & Stager & $168(70 \%)$ & $86.08 \pm 15.24$ & \multirow{2}{*}{5838} & $93.75 \pm 15.06$ & \multirow{2}{*}{6002} & $4.25 \pm 1.78$ & \multirow{2}{*}{$4994^{*}$} \\
\hline & Intern & $72(30 \%)$ & $86.57 \pm 15.92$ & & $94.29 \pm 15.26$ & & $3.68 \pm 1.69$ & \\
\hline \multirow{2}{*}{ Ward } & ER & $36(15 \%)$ & $90.22 \pm 13.16$ & \multirow{2}{*}{3262} & $94.44 \pm 13.41$ & \multirow{2}{*}{3529} & $3.44 \pm 1.73$ & \multirow{2}{*}{$2711^{* *}$} \\
\hline & Non-ER & $204(85 \%)$ & $85.52 \pm 15.70$ & & $93.81 \pm 15.40$ & & $4.19 \pm 1.76$ & \\
\hline \multirow{2}{*}{$\begin{array}{l}\text { Received education } \\
\text { about COVID-19? }\end{array}$} & Yes & $104(43.3 \%)$ & $86.82 \pm 15.36$ & \multirow{2}{*}{6673} & $94.93 \pm 11.93$ & \multirow{2}{*}{6932} & $4.15 \pm 1.85$ & \multirow{2}{*}{6879} \\
\hline & No & $136(56.7 \%)$ & $85.77 \pm 15.50$ & & $93.14 \pm 17.11$ & & $4.02 \pm 1.71$ & \\
\hline \multirow{4}{*}{ Source of education ${ }^{\mathrm{a}}$} & WHO, CDC, UpToDate & $41(17.1 \%)$ & $88.60 \pm 12.67$ & \multirow{4}{*}{293.5} & $95.83 \pm 9.31$ & & $3.95 \pm 1.82$ & \multirow{4}{*}{347} \\
\hline & National guidelines & $18(7.5 \%)$ & $79.49 \pm 19.26$ & & $95.68 \pm 13.28$ & 2305 & $3.72 \pm 1.74$ & \\
\hline & Both & $27(11.3 \%)$ & $87.32 \pm 14.73$ & & $97.12 \pm 7.91$ & 339.3 & $4.63 \pm 1.88$ & \\
\hline & Others & $18(7.5 \%)$ & $89.32 \pm 16.66$ & & $88.89 \pm 18.28$ & & $4.33 \pm 1.97$ & \\
\hline Years of age & \multicolumn{8}{|c|}{ Mean \pm SD $($ range $)=23.67 \pm 1.57(20-30)$} \\
\hline
\end{tabular}


Table 2. Level of COVID-19 Related Knowledge

\begin{tabular}{|c|c|}
\hline Items (True or False) & $\begin{array}{c}\text { Correct Answer Rate } \\
\text { (Range } 0-100 \%)\end{array}$ \\
\hline COVID-19 is a respiratory infection caused by a new species of coronavirus family. (T) & $95.4 \%$ \\
\hline The first case of COVID-19 was diagnosed in Wuhan, China. (T) & $91.7 \%$ \\
\hline The origin of COVID-19 is not clear but it seems that it has been transmitted to human by sea foods, snakes or bats. (T) & $82.5 \%$ \\
\hline Its common symptoms are fever, cough and shortness of breath but nausea and diarrhea were reported rarely. (T) & $90 \%$ \\
\hline Its incubation period is up to 14 days with a mean of 5 days. (T) & $85.4 \%$ \\
\hline $\begin{array}{l}\text { It can be diagnosed by PCR test on samples collected from nasopharyngeal and oropharyngeal discharge or from sputum and } \\
\text { bronchial washing. (T) }\end{array}$ & $80 \%$ \\
\hline It is transmitted through respiratory droplets such as cough and sneeze. (T) & $92.9 \%$ \\
\hline It is transmitted through close contacts with an infected case (especially in family, crowded places and health centers). (T) & $89.6 \%$ \\
\hline The disease can be prevented through handwashing and personal hygiene. (T) & $93.8 \%$ \\
\hline A medical mask is useful to prevent the spread of respiratory droplets during coughing. (T) & $85.5 \%$ \\
\hline $\begin{array}{l}\text { The disease can be prevented through no close contacts such as handshakes or kissing, not attending meetings and frequent hand } \\
\text { disinfection. (T) }\end{array}$ & $93.8 \%$ \\
\hline All people in society should wear masks. (F) & $82.1 \%$ \\
\hline Only during intubation, suction, bronchoscopy and cardiopulmonary resuscitation, you have to wear N95 mask. (T) & $79.6 \% *$ \\
\hline The disease can be treated by usual antiviral drugs. (F) & $88.3 \%$ \\
\hline $\begin{array}{l}\text { If symptoms appear within } 14 \text { days from direct contact with a suspected case, the person should inquire at a nearby public health } \\
\text { center. (T) }\end{array}$ & $73.8 \% *$ \\
\hline Total & $86.96 \%$ \\
\hline
\end{tabular}

T, True; F, False; COVID-19, Corona Virus Disease 2019.

*Items with lowest scores.

Table 3. Practicing Preventive Behaviors

\begin{tabular}{|c|c|}
\hline Items (Yes or No) & $\%$ of Yes \\
\hline I cancelled or postponed meetings with friends, eating-out and sport events. & $92.5 \%$ \\
\hline I reduced the use of public transportation. & $93.8 \%$ \\
\hline I went shopping less frequently. & $95 \%$ \\
\hline I reduced the use of closed spaces, such as library, theaters and cinema. & $94.6 \%$ \\
\hline I avoided coughing around people as much as possible. & $97.1 \%$ \\
\hline I avoided places where a large number of people are gathered. & $99.6 \%$ \\
\hline I increased the frequency of cleaning and disinfecting items that can be easily touched with hands (i.e. door handles and surfaces). & $85.6 \% *$ \\
\hline I washed the hands more often than usual. & $96.7 \%$ \\
\hline I discussed COVID-19 prevention with my family and friends. & $95.4 \%$ \\
\hline Total & $94.47 \%$ \\
\hline
\end{tabular}

Corona Virus Disease 2019.

*Items with lowest scores.

In this section, $30.8 \%$ of participants had high, $32.9 \%$ had moderate and $36.3 \%$ had low level of risk perception.

The correlation coefficient between major variables was also computed. Self-reported preventive behaviors and risk perception had a significantly negative correlation. ( $\mathrm{rs}=$ -0.128; $P<0.05$, Table 5).

\section{Discussion}

Since the novel corona virus has been discovered and started its journey around the world and the WHO declared the disease as a Public Health Emergency of International Concern, ${ }^{15}$ there has been great fear among all people about the possibility of a pandemic. ${ }^{16,17}$ Health professionals are no exception. Health professionals, especially medical students, who are the mainstay of health care workers in university hospitals, have always been at risk of infectious diseases, and the spread of the novel virus has increased the risk several times. Therefore, it is important to assess their knowledge about the nature of the virus, preventive behaviors and understanding their risk perception and anxiety level. With such information, health policy makers can make the proper planning.

According to our search, this is the first descriptive study in this field among medical students since the COVID-19 outbreak. In this study, which was conducted one week after the first diagnosis of the disease in Iran, we attempted to investigate the level of related knowledge, self-reported preventive behaviors and risk perception among medical students as well as the correlation between these major variables.

In this study, we found that $79.6 \%$ of medical students who participated in this survey had high level of related knowledge which was higher than previous studies conducted on health care workers. ${ }^{14,18}$ Among 
Table 4. Risk Perception of COVID-19

\begin{tabular}{lc}
\hline Items & Mean \pm SD (Possible Range: 1-4) \\
\hline I may become infected with COVID-19 more easily than others. & $2.04 \pm 0.96$ \\
I am afraid to be infected with COVID-19 & $2.04 \pm 0.97$ \\
Total & $4.08 \pm 1.77$ (possible range=2-8) \\
\hline
\end{tabular}

M, Mean; SD, standard deviation.

Table 5. Correlation between COVID-Related Knowledge, Preventive Behavior and Risk Perception among Iranian Medical Students

\begin{tabular}{lccc}
\hline Variables & Related-Knowledge & Preventive Behaviors & Risk Perception \\
\hline Related-knowledge & 1 & - & 1 \\
Preventive behaviors & -0.036 & - & - \\
Risk perception & 0.028 & $-0.128^{*}$ & 1 \\
\hline
\end{tabular}

* Significant at level of $P<0.05$; computed by Spearman rank correlation.

the participants, $94.2 \%$ had high level of performance in preventive behaviors which was higher than a study performed on health care workers. ${ }^{18}$ Risk perception among them was 4.08 out of 8 which was in the moderate range.

Due to lack of related literature, we compared the results of the present study with previous ones about MERS.

In the discussion of COVID-19 related knowledge items, the average of correct answers was $86.96 \%$, which was higher than the previous studies ${ }^{13,14}$ and similar to another study which reported $87.1 \% .{ }^{18}$ Two items with the lowest scores are worth mentioning. 'If symptoms appear within 14 days from direct contact with a suspected case, the person should inquire at a nearby public health center' with $73.8 \%$ which is important for referral of suspicious cases; and 'Only during intubation, suction, bronchoscopy and cardiopulmonary resuscitation, you have to wear N95 mask' with $79.6 \%$ which is about special self-protection for medical professionals. Therefore, for medical students who are expected to become general practitioners in the near future, more courses about case referrals and selfprotection should be included in their training programs.

The average score of preventive behaviors (94.47\%) was higher than a similar study results regarding MERS Coronavirus infection in Saudi Arabia. ${ }^{18}$ It is important to note that in its recommendations, the Iran's Ministry of Health has invited people to stay at home and not leave home during the first week of the outbreak. The Ministry has also focused on preventive education in a wide range. The results shown in Table 3 confirm this issue. However, the following item had the lowest score; 'I increased the frequency of cleaning and disinfecting items that can be easily touched with hands (i.e. door handles and surfaces)'. This item specifically outlines disinfection of surfaces and should be given more attention in future public education.

Both stagers and interns are trained in different parts of university hospitals. Interns, because they are in the 7 th year of studying medicine and have spent more time in clinical departments, are far more experienced than stagers. In this study, there was a significant difference between stagers and interns in risk perception. It can be inferred that the interns' greater experience and higher self-confidence in caring for patients have led them to perceive lower risk and experience less stress and anxiety.

In Iranian university hospitals, only interns, and not stagers, are in emergency departments and visit the patients. A minority (15\%) of participants were working in emergency departments at the time of the study. There was a significant difference between the risk perception of this group and those who were in non-emergency departments and wards. The personal protective equipment (such as special gowns, glasses for eye protection and N95 masks) was provided much more to emergency medical staff during the outbreak and provided greater safety, while other departments and wards were provided with ordinary protective equipment such as medical masks. It might be possible that adequate protective equipment suitable for the epidemic conditions can be effective in reducing stress and anxiety among health care workers.

According to the results of the study, there was a significant difference between males and females in risk perception, with risk perception being lower among females. This could possibly indicate that the women in this study were more aware of the importance of COVID-19.

The most interesting finding of this study is a negative significant correlation between self-reported preventive behaviors and risk perception. As preventive behaviors increase, risk perception declines. This finding is in contrast to the results of the study conducted by Rubin et al in which the two variables were concordant. ${ }^{19}$ In the aforementioned study, the participants were sampled from the general population and higher risk perception was associated with higher levels of preventive behaviors. However, in the present study, we investigated medical students who would know the consequences of preventive behaviors. Thus, negative correlation could be reasonable.

Therefore, to reduce risk perception, stress and anxiety which are major problems in disease outbreaks, ${ }^{20,21}$ preventive behavioral training should be increased among 
health care workers.

We believe that a limitation of this study was the risk perception section which had only two items and can be improved in future studies.

The most important problem we encountered during this outbreak was lack of pre-planned measures to evaluate the level of related knowledge and level of anxiety among health care workers. The present study can be a preliminary research and the results can be used by the health policy makers to focus on effective risk communication and education for the control of epidemics, especially for vulnerable groups like medical students.

In conclusion, based on our results, Iranian medical students had a high level of related knowledge and high performance in preventive behaviors, but a moderate risk perception. The negative correlation between self-reported preventive behaviors and risk perception was the most interesting finding in this study. In spite of high selfreported preventive behaviors, a moderate COVID-19 risk perception among medical students can raise concerns about their self-protective behaviors. As a vulnerable group, they must take the issue more seriously.

\section{Authors' Contribution}

MHT conceived the presented idea, reviewed the literature, and drafted the manuscript; RS supervised the project, reviewed the literature and contributed to manuscript drafting; RB gathered and analyzed the data and contributed to drafting the manuscript.

\section{Conflict of Interest Disclosure}

We know of no conflicts of interest associated with this publication and there has been no significant financial support for this work that could have influenced its outcome.

\section{Ethical Statement}

All participants completed the questionnaires with informed consent.

\section{Acknowledgements}

We feel obliged to thank all medical staff who truly dedicate their lives to fighting against COVID-19.

\section{References}

1. Zhu N, Zhang D, Wang W, Li X, Yang B, Song J, et al. A novel coronavirus from patients with pneumonia in China, 2019. N Engl J Med. 2020;382(8):727-733. doi: 10.1056/ NEJMoa2001017.

2. Habibzadeh P, Stoneman EK. The Novel Coronavirus: A Bird's Eye View. Int J Occup Environ Med. 2020;11(2):65-71. doi: 10.15171/ijoem.2020.1921.

3. Gorbalenya AE. Severe acute respiratory syndrome-related coronavirus-The species and its viruses, a statement of the Coronavirus Study Group. BioRxiv; 2020. doi: oi.o $\mathrm{rg} / 10.1101 / 2020.02 .07 .937862$

4. Lai CC, Shih TP, Ko WC, Tang HJ, Hsueh PR. Severe acute respiratory syndrome coronavirus 2 (SARS-CoV-2) and corona virus disease-2019 (COVID-19): the epidemic and the challenges. Int J Antimicrob Agents. 2020;55(3):105924. doi: 10.1016/j.ijantimicag.2020.105924.

5. Huang C, Wang Y, Li X, Ren L, Zhao J, Hu Y, et al. Clinical features of patients infected with 2019 novel coronavirus in Wuhan, China. Lancet. 2020;395(10223):497-506. doi: 10.1016/S0140-6736(20)30183-5.

6. Kenneth McIntosh M. Coronavirus disease 2019 (COVID-19). Available from: https://www.uptodate.com/contents/126981. Updated February 24, 2020.

7. Jahanpoor K. WEBDA, news station of ministry of health. Available from: http://air.ir/Zwq1Sao.

8. Raisei A. WEBDA, news station of ministry of health. Available from: http:// air.ir/ZwqbFao.

9. Biscayart C, Angeleri P, Lloveras S, Chaves T, Schlagenhauf $\mathrm{P}$, Rodríguez-Morales AJ. The next big threat to global health? 2019 novel coronavirus (2019-nCoV): What advice can we give to travellers? Interim recommendations January 2020, from the Latin-American society for Travel Medicine (SLAMVI). Travel Med Infect Dis. 2020;33:101567. doi: 10.1016/j. tmaid.2020.101567.

10. Bai Y, Yao L, Wei T, Tian F, Jin DY, Chen L, et al. Presumed Asymptomatic Carrier Transmission of COVID-19. JAMA. 2020 Feb 21. doi: 10.1001/jama.2020.2565.

11. Wang D, Hu B, Hu C, Zhu F, Liu X, Zhang J, et al. Clinical characteristics of 138 hospitalized patients with 2019 novel coronavirus-infected pneumonia in Wuhan, China. JAMA. 2020 Feb 7. doi: 10.1001/jama.2020.1585.

12. Chang D, Xu H, Rebaza A, Sharma L, Cruz CSD. Protecting health-care workers from subclinical coronavirus infection. Lancet Respir Med. 2020;8(3):e13. doi: 10.1016/S22132600(20)30066-7.

13. Kim JS, Choi JS. Middle East respiratory syndrome-related knowledge, preventive behaviours and risk perception among nursing students during outbreak. J Clin Nurs. 2016;25(1718):2542-9. doi: 10.1111/jocn. 13295 .

14. Khan MU, Shah S, Ahmad A, Fatokun O. Knowledge and attitude of healthcare workers about middle east respiratory syndrome in multispecialty hospitals of Qassim, Saudi Arabia. BMC Public Health. 2014;14:1281. doi: 10.1186/1471-245814-1281.

15. WHO. Statement on the second meeting of the International Health Regulations (2005) Emergency Committee regarding the outbreak of novel coronavirus (2019-nCoV). Available from: https://www.who.int/news-room/detail/30-01-2020statement-on-the-second-meeting-of-the-international-healthregulations-(2005)-emergency-committee-regarding-theoutbreak-of-novel-coronavirus-(2019-ncov).

16. Day M. Covid-19: surge in cases in Italy and South Korea makes pandemic look more likely. BMJ. 2020;368:m751. doi: 10.1136/bmj.m751.

17. Depoux A, Martin S, Karafillakis E, BSD RP, Wilder-Smith A, Larson $\mathrm{H}$. The pandemic of social media panic travels faster than the COVID-19 outbreak. J Travel Med. 2020 Mar 3. doi: 10.1093/jtm/taaa031.

18. Nour $\mathrm{MO}$, Babilghith $\mathrm{AO}$, Natto $\mathrm{HA}, \mathrm{Al}-\mathrm{Amin} \mathrm{FO}$, Alawneh SM. Knowledge, attitude and practices of healthcare providers towards MERS-CoV infection at Makkah hospitals, KSA. Int Res J Med Med Sci. 2015;3(4):103-12.

19. Rubin GJ, Amlôt R, Page L, Wessely S. Public perceptions, anxiety, and behaviour change in relation to the swine flu outbreak: cross sectional telephone survey. BMJ. 2009;339:b2651. doi: 10.1136/bmj.b2651.

20. Wong TW, Gao Y, Tam WWS. Anxiety among university students during the SARS epidemic in Hong Kong. Stress and Health: Journal of the International Society for the Investigation of Stress. 2007;23(1):31-5. doi: 0.1002/smi.1116

21. Cheong D, Lee C. Impact of severe acute respiratory syndrome on anxiety levels of front-line health care workers. Hong Kong Med J. 2004;10(5):325-30. 